\title{
SECOND ORDER NONLINEAR OPTICAL PROCESSES IN [111] CUBIC CRYSTALS FOR TERAHERTZ OPTOELECTRONICS
}

\author{
G. Gaborit, F. Sanjuan, and J.-L. Coutaz \\ IMEP-LAHC, University Savoie Mont-Blanc, 73763 Le Bourget du Lac Cedex, France \\ Email: coutaz@univ-savoie.fr
}

Received 12 February 2018; accepted 22 March 2018

\begin{abstract}
In this paper, we give an overview of emission and detection of terahertz electromagnetic pulses using, respectively, optical rectification and electro-optic effect in [111] zinc blende crystals. This crystal orientation allows us to generate and read any polarization state of the $\mathrm{THz}$ beam only by controlling the polarization state of the laser beam that excites or probes the emitting and receiving crystals. This technique is very useful for polarimetric terahertz spectroscopic studies.
\end{abstract}

Keywords: THz optoelectronics

PACS: $85.60 .-\mathrm{q}$

\section{Introduction}

While studied since the pioneering works of Bose [1], Rubens [2] and Nichols [3], researches in the far-infrared region of the electromagnetic spectrum, known today as the terahertz $(\mathrm{THz})$ domain, have been strongly boosted in the 80 's by the development of time-domain opto-electronic techniques [4] that were based on the use of reliable and efficient newly-invented mode-locked solid-state lasers. These lasers deliver extremely stable combs of femtosecond pulses. By rectifying such optical pulses in a nonlinear material or component, sub-picosecond electromagnetic bursts are generated, whose spectrum spreads typically from $100 \mathrm{GHz}$ up to a few THz. Time-resolved optoelectronic sampling techniques make it possible to record the burst waveforms with a subpicosecond time-equivalent resolution, thanks to the periodicity and repeatability of the laser pulses. The frequency associated with these $\mathrm{THz}$ pulses is obtained by a numerical Fourier transform. This method has allowed the development of the widely spread $\mathrm{THz}$ time-domain spectros- copy (TDS). Most of the materials and devices exhibiting a $\mathrm{THz}$ response have been studied so far. Today, a strong effort is done for achieving ultrabroad spectra, extending up to several tens of $\mathrm{THz}$ and even over $100 \mathrm{THz}$ [5], i.e. covering not only the far-infrared region, but also the mid-infrared and even the near-infrared. Simultaneously, researchers are aiming at producing extremely high power $\mathrm{THz}$ pulses, which allow one to perform nonlinear optics at $\mathrm{THz}$ frequencies, opening the route towards new topics in physics [6].

The key components in pulsed $\mathrm{THz}$ set-ups are the emitting and receiving antennas. They must efficiently rectify the femtosecond laser pulses without a too large broadening effect. Besides, in detection, the time-response should be as short as possible. This is mainly achieved with photoconducting switches, made with ultrafast semiconductor materials like low-temperature grown GaAs [7], or with electro-optic (EO) crystals [8].

This review paper is devoted to the $\mathrm{EO} \mathrm{THz}$ antennas. When illuminated by a powerful pulsed laser beam, optical rectification (OR) occurs in the crystals; more rigorously speaking, this 
phenomenon corresponds to difference-frequency generation, i.e. $\Omega=\omega_{1}-\omega_{2}$, where $\Omega$ is the $\mathrm{THz}$ frequency, and $\omega_{1}$ and $\omega_{2}$ are two frequencies belonging to a wide spectrum of the ultra-short laser pulses. Because $\Omega \ll \omega_{1}, \omega_{2}$, this frequency difference is approximated by $\mathrm{OR}$, i.e. $\omega_{1}-\omega_{2} \approx 0$. Efficient OR requires crystals exhibiting a large nonlinearity, and also phase-matching should be achieved, i.e. the laser group velocity and the $\mathrm{THz}$ phase velocity should be equal. Under these conditions, ultra broadband and high peak power $\mathrm{THz}$ signals are produced, thanks, respectively, to the almost instantaneous response of $\mathrm{OR}$, and to the possibility of exciting the crystals with intense laser pulses. In the receiving device, the signal is measured through the EO (Pockels) effect: the incoming $\mathrm{THz}$ field modifies the index ellipsoid of the crystal. The resulting induced birefringence is read with a laser probe beam. Here again, rigorously speaking, the involved phenomenon is difference-frequency generation $\omega_{1}+\Omega=\omega_{2}$ : to our knowledge, this induced change of frequency (up-conversion) has only been exploited by Minamide et al. [9] to read the $\mathrm{THz}$ signals. Highly nonlinear crystals for $\mathrm{THz}$ generation include ferroelectrics like $\mathrm{LiNbO}_{3}$,10], and organic crystals like DAST [11], while phasematching is almost achieved around $\lambda=800 \mathrm{~nm}$ in ZnTe [12], or in DAST around $\lambda=1.5 \mu \mathrm{m}$ [13].

Among these crystals, cubic crystals present the advantage of being isotropic when dealing with linear optics, which facilitates the arrangement of $\mathrm{THz}$ time-domain set-ups. Additionally, a variation of the crystal temperature does not influence the phase change induced by the THz field. Moreover, the second order nonlinear tensor involved either in OR or EO effects exhibits only a few nonnull elements, namely $\chi_{i j k}^{(2)} \equiv \chi_{14}(i \neq j \neq k)$, which simplifies the modeling of $\mathrm{THz}$ generation.

Usually, the crystals are cut in the [110] direction and are illuminated under normal incidence, which leads to the highest nonlinear efficiency. However, the [111] cut is very interesting, because it allows one to control the polarization of the generated $\mathrm{THz}$ beam by adjusting the one of the laser pump beam, and, in receiving antennas, the detection sensitivity is independent of the $\mathrm{THz}$ beam polarization. This enables one to manipulate the THz polarization in TDS set-ups, without using $\mathrm{THz}$ wave plates that are still difficult to design for broadband $\mathrm{THz}$ signals.
In this paper, we will give some basic properties of nonlinear optics with [111] cubic crystals. We will review $\mathrm{THz}$ emission by $\mathrm{OR}$ and $\mathrm{THz}$ detection by EO sampling, and we will finally address the limiting phenomenon of two-photon absorption (TPA).

\section{Nonlinear second and third order effects in cubic crystals}

\subsection{Basic concepts}

We define by $x, y, z$ and $X, Y, Z$ the frames, respectively, in the laboratory and in the crystal ( $X, Y, Z$ correspond to the crystallographic axes of the cubic crystal. From now on, capital letters refer to the crystal frame, while lower-cases are related to the laboratory frame). The laser beams (pump beam in the emitter and probe beam in the receiver) propagate in the laboratory along the axis $z$. We set $\omega$ and $\Omega$ the frequencies of, respectively, the laser beam and the $\mathrm{THz}$ beam.

Nonlinear tensors are usually defined in the frame of the crystal. Thus, calculations should be performed as follows: first, the incoming fields (laser in the OR case, laser and $\mathrm{THz}$ in EO sampling) are written in the crystal frame by applying a rotation matrix $R$. Then the nonlinear effect is calculated. The resulting fields are obtained in the crystal frame and are calculated in the laboratory frame by using the inverse matrix $R^{-1}$. This procedure is compulsory as all the quantities are measured in the laboratory frame.

To go from the laboratory $x, y, z$ frame to the crystal $X, Y, Z$ frame, a first rotation by $\theta$ around axis $y$ is performed, leading to new axes $x^{\prime}, y, z^{\prime}$, followed by a second one by $\varphi$ around $z$. The expression of the rotation matrix is

$$
\begin{aligned}
& R(\varphi, \theta)=R(\varphi) R(\theta) \\
& =\left(\begin{array}{ccc}
\cos \theta \cos \varphi & -\sin \varphi & \cos \theta \\
\cos \theta \sin \varphi & \cos \varphi & \sin \theta \sin \varphi \\
-\sin \theta & 0 & \cos \theta
\end{array}\right) .
\end{aligned}
$$

In cubic crystals, the nonlinear second and third order tensors take the following forms

$$
\chi_{I J K}^{(2)}=\chi_{14}^{(2)} \delta_{I J} \delta_{I K^{\prime}}
$$




$$
\begin{aligned}
& \chi_{I J K L}^{(3)}=\chi_{1}^{(3)} \delta_{I J} \delta_{I K} \delta_{K L}+\chi_{2}^{(3)} \delta_{I J} \delta_{K L} \\
& +\chi_{3}^{(3)} \delta_{I K} \delta_{I L}+\chi_{4}^{(3)} \delta_{I L} \delta_{I K}
\end{aligned}
$$

where $\delta_{I J}$ is the Kronecker symbol $\left(\delta_{I I}=1, \delta_{I \neq J}=0\right)$. The second order tensor describes OR and EO, while the third order one is related to TPA. Because TPA involves laser photons at the same frequency $\omega$ (see Eqs. (1) and (2)), thus $\chi_{2}^{(3)}=\chi_{3}^{(3)}$.

\subsection{Generation of $\mathrm{THz}$ by OR in cubic crystals}

In the undepleted pump beam approximation, the propagation equation of the laser beam field does not depend on the $\mathrm{THz}$ field. Therefore, the $\mathrm{THz}$ field obeys only the following differential equation

$$
\begin{aligned}
& \frac{\partial^{2} \vec{E}_{\Omega}}{\partial z^{2}}+\widetilde{k}_{\Omega}^{2} \vec{E}_{\Omega} \\
& =-\frac{\Omega^{2}}{c^{2}} \vec{\chi}^{(2)}(\Omega, \omega+\Omega,-\omega): \vec{E}_{\omega+\Omega} \cdot \vec{E}_{\omega,}^{*}
\end{aligned}
$$

where $\widetilde{k}_{\Omega}^{2}=\widetilde{\varepsilon}_{\Omega} \Omega^{2} / c^{2}, \widetilde{\varepsilon}_{\Omega}$ is the dielectric constant at $\mathrm{THz}$ frequency ${ }^{1}$, and we do not write the $z$ dependence of the laser field for the sake of simplicity. Also we do not take into account TPA effects: TPA will be treated in the last part of this paper. The generated $\mathrm{THz}$ field is thus proportional to the nonlinear term in (4). As already explained, this term must be calculated in the crystal frame and then in the laboratory frame.

We start from the pump laser field that, in the laboratory, is polarized in the $x y$ plane, i.e.

$$
\vec{E}_{\omega, \mathrm{lab}}=E_{\omega, \mathrm{lab}}\left(\begin{array}{c}
\cos \psi \\
\sin \psi \\
0
\end{array}\right),
$$

where $\psi$ is the angle of the laser beam polarization in the $x y$ plane. Then we calculate the nonlinear polarization in the laboratory frame:

$$
\begin{aligned}
& \vec{P}_{\text {lab }}^{N L}=R(-\varphi,-\theta) \\
& \times\left(\varepsilon_{0} \vec{\chi}^{(2)}: R(\theta, \varphi) \vec{E}_{\omega, \text { lab }} \cdot R(\theta, \varphi) \vec{E}_{\omega, \text { lab }}\right) .
\end{aligned}
$$

${ }^{1}$ In this paper, a complex value is pointed out by a tilde, but neither the vectors nor the tensors with a complex amplitude or complex elements.
Therefore, in the referential laboratory, the $\mathrm{THz}$ field is

$$
\begin{aligned}
& \vec{E}_{\Omega,(x, y, z)}= \\
& \left(\begin{array}{c}
E_{\Omega, X} \cos \varphi \cos \theta+E_{\Omega, Y} \sin \varphi \cos \theta+E_{\Omega, Z} \sin \theta \\
-E_{\Omega, X} \sin \varphi+E_{\Omega, Y} \cos \varphi \\
-E_{\Omega, X} \sin \theta \cos \varphi-E_{\Omega, Y} \sin \theta \sin \varphi+E_{\Omega, Z} \cos \theta
\end{array}\right)
\end{aligned}
$$

where $E_{\Omega,(X, Y, Z)}$ are the components of the $\mathrm{THz}$ generated field in the crystal frame, whose expressions are

$$
\begin{aligned}
& \vec{E}_{\Omega,(X, Y, Z)} \propto \vec{\chi}^{(2)}(\Omega, \omega+\Omega,-\omega): \vec{E}_{\omega+\Omega} \vec{E}_{\omega}^{*} \\
& \propto \chi_{14}^{(2)} E_{\omega}^{2} \\
& \times\left(\begin{array}{l}
(\cos \psi \cos \theta \sin \varphi+\sin \psi \cos \varphi) \cos \psi \sin \theta \\
(\cos \psi \cos \theta \cos \varphi-\sin \psi \sin \varphi) \cos \psi \sin \theta \\
(\cos \psi \cos \theta \sin \varphi+\sin \psi \cos \varphi)(\cos \psi \cos \theta \cos \varphi \\
-\sin \psi \sin \varphi)
\end{array}\right) .
\end{aligned}
$$

It is useful to derive the intensity of the $\mathrm{THz}$ beam from (8):

$$
\begin{aligned}
& I_{\Omega} \propto E_{\Omega,(x, y, z)}^{2} \\
& \propto\left(\chi_{14}^{(2)} I_{\omega}\right)^{2}\left[\left(\begin{array}{c}
(\cos \theta \cos \varphi \cos \psi-\sin \varphi \sin \psi) \\
\times(\cos \theta \sin \varphi \cos \psi+\cos \varphi \sin \psi) \\
+\sin ^{2} \theta \cos ^{2} \psi\left(1-\sin ^{2} \theta \cos ^{2} \psi\right)
\end{array}\right)^{2}\right] .
\end{aligned}
$$

$I_{\Omega}$ does not depend on the laser beam polarization angle $\psi$ only if $\cos \theta=1 / \sqrt{3}$ and $\varphi=\pi / 4$, which corresponds to the [111] cut.

Table 1. Parameters for THz emission through OR in cubic crystals with different cuts.

\begin{tabular}{c|c|c|c}
\hline Crystal cut & {$[100]$} & {$[110]$} & {$[111]$} \\
\hline Angle $\varphi$ & 0 & $\pi / 4$ & $\pi / 4$ \\
\hline Angle $\theta$ & $\pi / 2$ & $\pi / 2$ & $\operatorname{acos}(1 / \sqrt{3})$ \\
\hline$E_{\Omega, x}$ & 0 & $(\cos 2 \psi-1) / 4$ & $\cos (2 \psi) / \sqrt{6}$ \\
\hline$E_{\Omega, y}$ & 0 & $-\sin 2 \psi / 2$ & $-\sin 2 \psi / \sqrt{6}$ \\
\hline$E_{\Omega, z}$ & $-\sin 2 \psi / 2$ & 0 & $-1 / 2 \sqrt{3}$ \\
\hline$I_{\Omega}$ & \multicolumn{4}{|}{} \\
\hline
\end{tabular}

Table 1 gives the THz field components radiated by cubic crystals of different cuts under a normal incidence excitation. When using the [111] cut, 
the $\mathrm{THz}$ field is linearly polarized and its polarization angle is $\psi_{\Omega}=-2 \psi$ (see Fig. 1 ), while the $\mathrm{THz}$ field magnitude is independent of $\psi$. Therefore, it is fully possible to monitor the radiated $\mathrm{THz}$ field polarization state by adjusting the laser polarization state that could be, for example, linear, circular, elliptical, and so on.

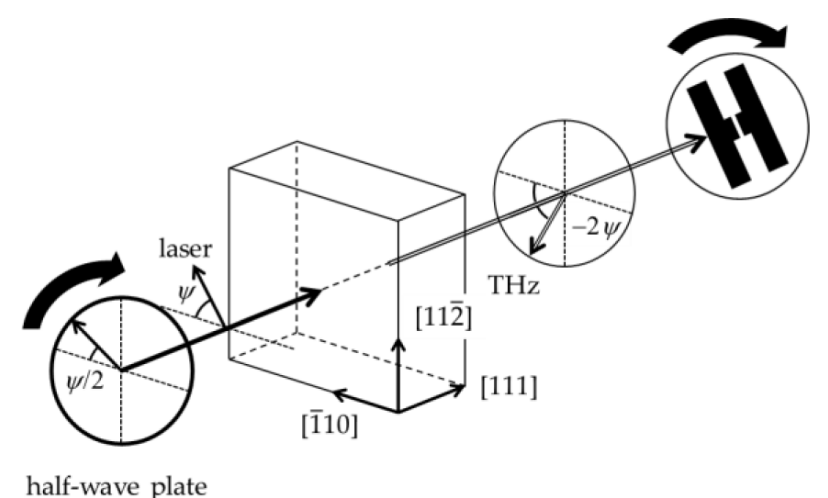

Fig. 1. Generation of a linearly polarized $\mathrm{THz}$ field by OR in a [111] cubic EO crystal.

Let us notice that the $\mathrm{THz}$ field longitudinal component, i.e. along $z$, exists in the crystal, but does not propagate in the surrounding medium, where the THz field has to be transverse. The [100]cut does not radiate $\mathrm{THz}$ wave, while the [110]-cut is delivering a strongly $\psi$-dependent signal. This is illustrated in Fig. 2 where $I_{\Omega}$ is plotted versus $\psi$ for different crystal cuts. As compared to the maximum $\mathrm{THz}$ intensity generated with a [110] crystal, the intensity with [111] ones is only $16 \%$ weaker.

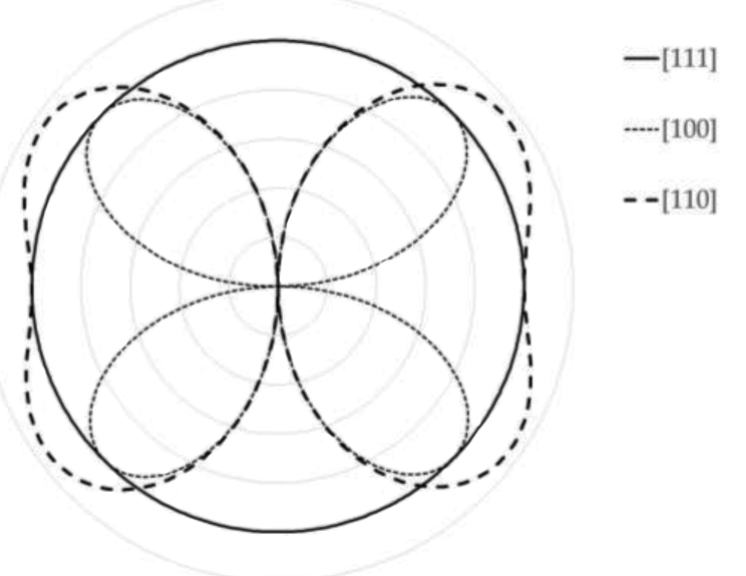

Fig. 2. THz intensity in the crystal versus the laser polarization angle $\psi$ for different orientations of a cubic crystal (polar plot).

\subsection{EO sampling with [111] cubic crystals}

In the detecting antenna, the nonlinear crystal is illuminated simultaneously by the $\mathrm{THz}$ pulse and the laser probe pulse. Through the Pockels effect, the $\mathrm{THz}$ field induces a birefringence in the crystal, which is read with the probe beam. Even if different detection schemes can be used [14], generally a polarimetric method is employed to measure the induced birefringence and then to deduce the $\mathrm{THz}$ field strength and polarization. In this case, a circularly-polarized probe beam propagates through the crystal, and it becomes elliptic because of the crystal birefringence. In cubic crystals, the birefringence exists only through the EO effect due to the impinging $\mathrm{THz}$ field, while in anisotropic crystals, the EO effect mostly changes weakly the length of the ellipsoid axes but not their directions. Therefore, the orientation of the EOinduced ellipsoid must be determined for cubic crystals. The elliptical polarization is transformed into a linear one by a quarter wave plate (QWP) located after the EO crystal, and the resulting linear polarization direction is measured with a polarizing prism, like a Wollaston one. Let us explain the main details of this detection scheme.

In the crystal frame, the ellipsoid equation is

$$
\frac{X^{2}+Y^{2}+Z^{2}}{n_{\omega}^{2}}+2 r_{14}\left(Y Z E_{\Omega X}+Z X E_{\Omega X}+X Y E_{\Omega Z}\right)=1 .
$$

This equation can be written as a matrix product

$$
\left(\begin{array}{lll}
X & Y & Z
\end{array}\right)\left(\begin{array}{ccc}
n_{\omega}^{-2} & A & C \\
A & n_{\omega}^{-2} & B \\
C & B & n_{\omega}^{-2}
\end{array}\right)\left(\begin{array}{l}
X \\
Y \\
Z
\end{array}\right)=1 \text {, }
$$

with

$$
A=r_{14} E_{\Omega Z}, B=r_{14} E_{\Omega X}, C=r_{14} E_{\Omega Y} .
$$

Calculating the eigenvalues and eigenvectors of the matrix $M_{\mathrm{EO}}$ in (11) is quite tricky and leads to very long and complicated expressions. In fact, one does not need to know the ellipsoid in the laboratory frame, but only its projection (ellipse) on a plane perpendicular to the laser beam is requested [15]. This greatly simplifies the calculation. Let us set $m_{\mathrm{EO}}$ the matrix in the laboratory frame:

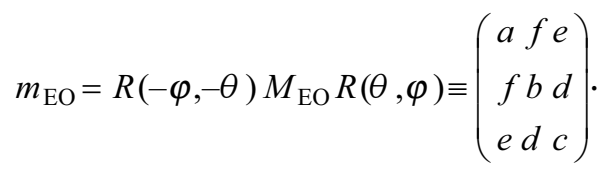


The ellipsoid equation is written in the laboratory frame and for $z=0$ (plane perpendicular to the laser beam):

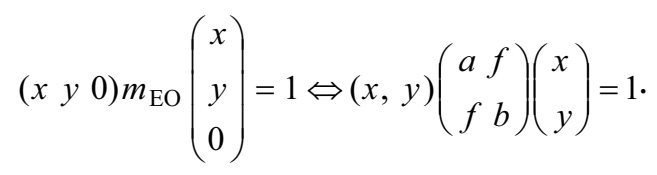

The eigenvalues and eigenvectors of this matrix are

$$
\begin{aligned}
& \lambda_{1,2}=\frac{a+b \pm \sqrt{(a-b)^{2}+4 f^{2}}}{2}, \\
& \vec{V}_{1,2}=\left(\begin{array}{c}
2 f \\
a-b \pm \sqrt{(a-b)^{2}+4 f^{2}}
\end{array}\right) .
\end{aligned}
$$

For different crystal cuts, we end up with Table 2 in which $\Delta n_{\omega}=n_{\omega}\left(E_{\Omega}\right)-n_{\omega}\left(E_{\Omega}=0\right)=n_{\omega}\left(E_{\Omega}\right)-n_{\omega}$ is obtained in the weak EO effect approximation $\left(\Delta n_{\omega} \ll n_{\omega}\right)$. This table is established in the case the laser and $\mathrm{THz}$ beams are collinear.

Therefore, the [100] cut is not suitable for EO detection under normal incidence; the [110] cut sensitivity is strongly dependent on the $\mathrm{THz}$ polarization. The [111] cut is highly attractive because the EO efficiency does only depend on the $\mathrm{THz}$ field strength $E_{\Omega}$ and not on its polarization. In this case, the induced index ellipse is oriented at $-\psi_{\Omega} / 2$ from the $x$ direction when the incident $\mathrm{THz}$ beam polarization angle is $\psi_{\Omega}$. This effect is the inverse of what we determined for OR emission that is oriented at $-2 \psi$ when the laser pump polarization angle is $\psi$.

Let us now explain how to measure the induced birefringence. We employ a laser probe beam with a circular polarization that is obtained with a QWP from the linearly polarized incoming laser beam. After passing through the EO crystal, the polarization state of the laser probe beam is elliptically polar- ized. The two perpendicular components of the $\mathrm{THz}$ field are separated by a Wollaston prism and directed towards a balanced two-photodiodes detection system. In front of the Wollaston prism, a half-wave plate (HWP) allows to rotate the elliptical polarization in view of optimizing the detected signal. Using a Jones matrix formalism, the laser field at the Wollaston prism is

$$
\begin{aligned}
& \vec{E}_{\omega, \text { det }}=R(-\alpha) M_{\mathrm{HWP}} R(\alpha) R\left(-\gamma+\frac{\psi_{\Omega}}{2}\right) \\
& \times M_{\mathrm{crys}} R\left(\gamma-\frac{\psi_{\Omega}}{2}-\frac{\pi}{4}\right) M_{\mathrm{QWP}} R\left(\frac{\pi}{4}\right) \vec{E}_{\omega} .
\end{aligned}
$$

Here $\gamma$ and $\alpha$ are the orientation angles of, respectively, the [112] axis of the EO crystal, and the fast axis of the HWP. $M_{\mathrm{QWP}} M_{\mathrm{HWP}}$ and $M_{\text {crys }}$ are the Jones matrices of the QWP, HWP and EO crystal ([111] cut). When the EO crystal is made birefringent by the incoming $\mathrm{THz}$ beam, $M_{\text {crys }}$ writes:

$$
M_{\text {crys }}=\mathrm{e}^{-\mathrm{i} \frac{\omega}{c} n_{o, \omega} d}\left(\begin{array}{cc}
\mathrm{e}^{-\mathrm{i} \eta E} & 0 \\
0 & \mathrm{e}^{\mathrm{i} \eta E_{\Omega}}
\end{array}\right), \eta=\frac{r_{14}}{\sqrt{6} n_{o, \omega}^{3} .}
$$

The signal delivered by the balanced photodiodes is proportional to the difference of the intensities of the two perpendicularly polarized beams separated by the Wollaston prism, i.e. [16]:

$$
S_{\text {photodiode }} \propto E_{\omega, \text { det }, x}^{2}-E_{\omega, \text { det } y}^{2} \propto E_{\Omega} \sin \left(3 \gamma+\psi_{\Omega}-4 \alpha\right) .(19
$$

Whatever is the polarization $\psi_{\Omega}$ of the incident

\begin{tabular}{|c|c|c|c|c|c|c|}
\hline & $\frac{a-1 / n_{\omega}^{2}}{r_{14}}$ & $\frac{b-1 / n_{\omega}^{2}}{r_{14}}$ & $\frac{f}{r_{14}}$ & $2 \frac{\lambda_{1,2}-1 / n_{\omega}^{2}}{r_{14}}$ & $\Delta n_{\omega}$ & $\vec{V}_{1}$ \\
\hline 100 & 0 & 0 & 0 & 0 & 0 & - \\
\hline 110 & 0 & $E_{\Omega x}$ & $E_{\Omega y}$ & $E_{\Omega_{x}} \pm \sqrt{E_{\Omega x}^{2}+4 E_{\Omega y}^{2}}$ & $-\frac{r_{14}}{4} n_{\omega}^{3}\left(E_{\Omega x} \pm \sqrt{E_{\Omega x}^{2}+4 E_{\Omega y}^{2}}\right.$ & $\left(\begin{array}{c}2 \sin \psi_{\Omega} \\
-\cos \psi_{\Omega}+\sqrt{1+3 \sin ^{2} \psi_{\Omega}}\end{array}\right.$ \\
\hline 111 & $-\sqrt{\frac{2}{3}} E_{\Omega x}$ & $\sqrt{\frac{2}{3}} E_{\Omega x}$ & $\sqrt{\frac{2}{3}} E_{\Omega y}$ & $\pm \sqrt{\frac{8}{3}} E_{\Omega}$ & $\pm \frac{r_{14}}{\sqrt{6}} n_{o, \omega}^{3} E_{\Omega}$ & $\left(\begin{array}{l}\cos \frac{\psi_{\Omega}}{2} \\
-\sin \frac{\psi_{\Omega}}{2}\end{array}\right)$ \\
\hline
\end{tabular}
$\mathrm{THz}$ beam and the orientation $\gamma$ of the EO crystal, there exists an optimal orientation $\alpha_{\text {opt }}$ of the HWP for which the detected signal $S_{\text {photodiode }}$ is maximum:

$$
\alpha_{\mathrm{opt}}=\frac{3 \gamma+\psi_{\Omega}}{4} .
$$

Table 2. Parameters for EO detection of THz fields in cubic crystals with different cuts. 
Therefore the [111] cut always allows one to perform an optimized detection of the $\mathrm{THz}$ field. However, rotating the HWP in front of the detector may be inconvenient. A smart scheme has been introduced by van der Valk et al. in view of performing polarization imaging [17]. The laser probe beam transmitted by the EO crystal is split in two beams and each of them is detected using the arrangement explained above (Wollaston prism and balanced photodiodes). In the second beam, a HWP is oriented at $22.5^{\circ}=\pi / 8$. This HWP rotates the elliptically polarized probe beam by $\pi / 4$. Here the detected fields in the first and second channels are

$$
\begin{aligned}
& \vec{E}_{\omega, \text { det }, 1}=R\left(-\gamma+\frac{\psi_{\Omega}}{2}\right) M_{\text {crys }} \\
& \times R\left(\gamma-\frac{\psi_{\Omega}}{2}-\frac{\pi}{4}\right) M_{\mathrm{QWP}} R\left(\frac{\pi}{4}\right) \vec{E}_{\omega}, \\
& \vec{E}_{\omega, \text { det }, 2}=R\left(-\frac{\pi}{8}\right)\left(\begin{array}{cc}
\mathrm{e}^{-\mathrm{i} \frac{\pi}{2}}-1 \\
1 & \mathrm{e}^{-\mathrm{i} \frac{\pi}{2}}
\end{array}\right) R\left(\frac{\pi}{8}\right) \vec{E}_{\omega, \text { det }, 1} .
\end{aligned}
$$

Performing the products in Eq. (21) leads to

$$
\begin{aligned}
& \left|\vec{E}_{\omega, \operatorname{det}, 1}\right|_{\left(\begin{array}{l}
x \\
y
\end{array}\right)}^{2} \approx \frac{1 \mp 2 \eta E_{\Omega} \sin \left(2 \gamma-\psi_{\Omega}\right)}{2} E_{\omega}^{2}, \\
& \left|\vec{E}_{\omega, \operatorname{det}, 2}\right|_{\left(\begin{array}{l}
x \\
y
\end{array}\right)}^{2} \approx \frac{1 \mp 2 \eta E_{\Omega} \cos \left(2 \gamma-\psi_{\Omega}\right)}{2} E_{\omega}^{2} .
\end{aligned}
$$

Therefore, the signals delivered by the first and second channels are, respectively,

$$
\begin{aligned}
& S_{1} \propto\left|\vec{E}_{\omega, \operatorname{det}, 1}\right|_{x}^{2}-\left|\vec{E}_{\omega, \operatorname{det}, 1}\right|_{y}^{2} \propto-E_{\Omega} \sin \left(2 \gamma-\psi_{\Omega}\right), \\
& S_{2} \propto E_{\Omega} \cos \left(2 \gamma-\psi_{\Omega}\right) .
\end{aligned}
$$

If the crystal axes are aligned with the laboratory frame, i.e. $\gamma=0$, the two detections channels give simultaneously the two components of the $\mathrm{THz}$ field:

$$
S_{2} \propto E_{\Omega} \sin \psi_{\Omega}=E_{\Omega y}, S_{2} \propto E_{\Omega} \cos \psi_{\Omega}=E_{\Omega x} .
$$

\subsection{Figure-of-merit of cubic EO crystals}

When TPA does not perturb the THz generation through the OR process, the propagation equation (4) has an analytic solution [11]:

$$
\begin{aligned}
& E_{\Omega}(z)=\frac{\mu_{o} \chi^{(2)} \Omega I_{\text {laser }}(\Omega)}{n_{o, \omega}\left[\frac{c}{\Omega}\left(\frac{\alpha_{\Omega}}{2}+\alpha_{\omega}\right)+\mathrm{i}\left(n_{\Omega}+n_{\mathrm{G} \omega}\right)\right]} \\
& \times \frac{\mathrm{e}^{-\mathrm{i} \frac{\Omega}{c} n_{\Omega} z} \mathrm{e}^{-\frac{\alpha_{\Omega}}{2} z}-\mathrm{e}^{-\mathrm{i} \frac{\Omega}{c} n_{\mathrm{G} \omega} z} \mathrm{e}^{-\alpha_{\omega} z}}{\frac{\alpha_{\Omega}}{2}-\alpha_{\omega}+\mathrm{i} \frac{\Omega}{c}\left(n_{\Omega}-n_{\mathrm{G} \omega}\right)} .
\end{aligned}
$$

$I_{\text {laser }}(\Omega)$ is the spectral component of the laser intensity that is separated by $\Omega$ from the laser central frequency. $n_{\mathrm{G} \omega}$ is the group index of the crystal at the laser frequency. When taking into account the transmission of the laser beam at the crystal entrance face, as well as the transmission of the $\mathrm{THz}$ field at the output face, one can show [11] that the $\mathrm{THz}$ field is

$$
I_{\Omega}(z) \propto \frac{4 n_{o, \omega}^{7} r_{i j}^{2} z^{2} I(\Omega)^{2}}{\left(1+n_{o, \omega}\right)^{2}\left(1+n_{\mathrm{G} \omega}\right)^{2}} \equiv F o M \times(z I(\Omega))^{2} .
$$

To obtain (28), absorption is neglected and phase matching is supposed to be almost fulfilled $\left(n_{\Omega} \approx n_{G \omega}\right)$. FoM is the factor of merit of nonlinear crystals for the OR process. It can also be used to select the best crystals for EO sampling, as OR and $\mathrm{EO}$ tensors are equal. Table 3 summarizes the main

Table 3. Main EO cubic crystals.

\begin{tabular}{cccccccccc}
\hline & $\lambda_{\text {laser }}$ & $n_{\omega}$ & $n_{\Omega}$ & $r_{14}$ & $F o M$ & $E_{\text {gap }}$ & $f_{\text {phonon }}$ \\
\hline & $\mu \mathrm{m}$ & & & $\mathrm{pm} / \mathrm{V}$ & $(\mathrm{pm} / \mathrm{V})^{2}$ & $\mathrm{eV}$ & $\mathrm{THz}$ \\
\hline $\mathrm{GaAs}$ & 0.8 & 3.33 & 3.6 & 1.3 & 86 & 1.42 & 8 \\
\hline $\mathrm{ZnTe}$ & 0.8 & 2.85 & 3.23 & 4 & 370 & 2.26 & 5.4 \\
\hline $\mathrm{CdTe}$ & 10.6 & 2.64 & 3.23 & 6.8 & 700 & 1.44 & 4.4 \\
\hline $\mathrm{ZnSe}$ & 0.8 & 2.52 & 3.00 & 2.0 & 52 & 2.70 & 6.2 \\
\hline $\mathrm{GaP}$ & 0.8 & 2.95 & 3.3 & 0.97 & 25 & 2.26 & 11 \\
\hline $\mathrm{ZnS}$ & 1 & 2.29 & 2.90 & 1.7 & 23 & 3.54 & 8.4 \\
\hline
\end{tabular}


properties of cubic crystals that can be used for OR generation and $\mathrm{EO}$ sampling of $\mathrm{THz}$ beams. Some crystals, like InAs or InP, are missing because they are mostly employed for $\mathrm{THz}$ generation through the surface acceleration of photo-carriers (namely the Dember effect [7]).

CdTe exhibits the best FoM among the listed crystals, however, its absorption at $\mathrm{THz}$ frequencies is not null because of a phonon resonance at 4.4 THz. Therefore, even if less efficient, ZnTe is widely used for both OR and $\mathrm{EO}$ detection of $\mathrm{THz}$ beams.

\section{Experimental works}

\subsection{State of the art in $\mathrm{THz}$ OR with zinc-blende [111] crystals}

Generation of the far-infrared signal by OR in nonlinear dielectric crystals was first proposed and demonstrated by Zernike and Berman in 1965 [18], and followed in the '60s by a few reports on the generation by optical frequency difference in such crystals [19-22]. However, the first report of far-infrared generation using ultra-short laser pulses (picosecond at those times) was published by Y.R. Shen and his team [23-24]. A. Bonvalet et al. [25] were the first to make use of a femtosecond laser, and they generated ultra-broad band signals spreading up to $50 \mathrm{THz}$ with a [110] zinc blende crystal, namely GaAs. Then X.-C. Zhang and his team made several seminal advances to employ OR in zinc-blende crystals as a source of $\mathrm{THz}$ radiation in time-domain systems (see, for example, [26, 27]). Several zinc blende crystals were investigated, like GaP, GaAs, ZnTe, etc. Even if generation in [111] crystal samples was reported, the polarization question was not addressed in these pioneering papers, because a large broadband and high power generation were the main goals of these researches.

To our knowledge, the team of T. Norris at the University of Michigan was the first one to generate $\mathrm{THz}$ pulses in [111] cubic crystals with a polarization state controlled by the one of the exciting laser beam [28]. In this work, the THz ellipticity was controlled by adjusting both the relative time delay in the nonlinear crystal of two exiting pulses delivered by two lasers, and the polarization between two lasers. A great improvement was demonstrated by the team of M. Kuwata-Gonokami in Japan [29], who employed a single femtosecond laser and derived the relation $\psi_{\Omega}=-2 \psi$ established in the first part of this paper. In [25], the [111] zinc blende crystal was GaP, and the detection of the $\mathrm{THz}$ signal was based on sum frequency generation. A great demonstration of polarization-shaped $\mathrm{THz}$ spectra was realized, and, for example, left- and right-circularly polarized $\mathrm{THz}$ pulses were produced. Later on, we published a general theory of $\mathrm{THz}$ generation through OR in cubic crystals, with a special emphasize on the choice of the crystal cut [30].

\subsection{State of the art in THz EO sampling with zinc-blende [111] crystals}

G. Mourou and his colleagues were the first to employ the EO sampling technique to measure waveforms of electrical transients propagating along microwaves lines [31]. Then the technique was applied to sample freely-propagating $\mathrm{THz}$ pulses [32-34]. Among the different crystals tested for EO sampling of $\mathrm{THz}$ pulse $\left(\mathrm{LiNbO}_{3}, \mathrm{LiTaO}_{3}\right.$, DAST...), zinc blende crystals like ZnTe and GaP attracted a great attention because of their isotropic behaviour and of the possibility of achieving ZnTe phase-matching between the incoming $\mathrm{THz}$ beam and the laser probe beam. Most of the publications made use of [110] cubic crystals that lead to the strongest signal in detection. However, the crystal axes should be oriented for each polarization of the $\mathrm{THz}$ beam in view of optimizing the detected signal. To solve this problem, an ultimate scheme includes an EO crystal that is continuously rotated in the $\mathrm{THz}$ beam during measurement [35].

Our team was the first to propose and theoretically demonstrate that the sensitivity of an EO detector made with a [111] zinc blende crystal is independent of the direction of the $\mathrm{THz}$ electric field polarization, and that they could detect $\mathrm{THz}$ pulses, whatever their polarization [15]. This was experimentally demonstrated by van der Valk et al. [17] who, in addition, introduced the smart EO detection scheme described above, with which measuring the polarization of the $\mathrm{THz}$ pulse is performed without rotating any component of the set-up. This system was employed to record polarized $\mathrm{THz}$ images. More recently [30], we derived and experimentally validated 
the practical analytic expressions that give the recorded signal versus the impinging $\mathrm{THz}$ field vector (magnitude and direction) and the orientation of the EO crystal axes and of the necessary wave-plates. Another polarimetric detection based on [111] zinc blende crystals was published in [36]: the direction of the $\mathrm{THz}$ electric field was obtained with another detection method using a [111] cubic crystal, based on a polarization modulation technique with an electro-optic modulator. Unfortunately, recording the signal is rather long and imprecise. Still using this polarization modulation technique, the polarization of linearly-polarized $\mathrm{THz}$ fields was obtained by rotating either a polarizer [37] or the [111]-cut crystal [38].

\subsection{Experimental demonstration of optically controlling the generated $\mathrm{THz}$ polarization}

In this paragraph, we show that the above relations (1)-(28) are nicely verified by experimental works, and thus [111] cubic crystals allow buildup of THz-TDS systems in which the $\mathrm{THz}$ polarization is only controlled in emission and detection through the laser polarization.

We choose ZnTe as the nonlinear crystal because its FoM is quite strong, even if its nonlinear coefficients $\left(r_{14}, \chi_{14}\right)$ are not the largest ones. The laser is either a Ti:sapphire mode-locked laser from Femtolasers, delivering 50 fs pulses at the sample location $(80-\mathrm{MHz}$ repetition rate, $200-\mathrm{mW}$ average power), or a Ti:sapphire amplified system from Coherent (Libra model) delivering 5-mJ, 45-fs pulses at a $1-\mathrm{kHz}$ repetition rate. Both lasers' wavelength is $\sim 800 \mathrm{~nm}$.
In a first experiment, we make use of a typical free space THz-TDS set-up. The THz pulse emission is performed with the [111] ZnTe crystal, in front of which a HWP allows us to adjust the angle of polarization of the impinging laser beam. The THz beam detection is realized with a LT-GaAs photo-conducting antenna from Teravil, which is sensitive to the $\mathrm{THz}$ polarization and which is mounted on a rotation stage. For each orientation of the receiver $\left(0\right.$ to $\left.110^{\circ}\right)$, the peak $\mathrm{THz}$ signal is recorded every $4^{\circ}$ from $0^{\circ}$ up to $180^{\circ}$. The result is presented in Fig. 3 .

As expected, the experimental data are well fitted by relation (19) and thus vary as $\sin \left(\psi_{\Omega}\right)=$ $\sin (2 \psi)=\sin \left(4 \psi_{\mathrm{HWP}}\right)$, where $\psi_{\mathrm{HWP}} \psi$, and $\psi_{\Omega}$ are the orientation/polarization angles of the HWP, of the laser beam and of the $\mathrm{THz}$ beam, respectively. Moreover, one can notice that for each $\mathrm{THz}$ beam polarization $\psi_{\Omega}$, one can adjust the orientation of the receiver in order to maximize the detected signal. Thus, a proper HWP orientation enables us to adjust the desired $\mathrm{THz}$ polarization, and a proper orientation of the EO receiving crystal allows us to maximize the detected signal. The orientation of the generated $\mathrm{THz}$ polarization is determined from Fig. 3 , and is plotted in Fig. 4 as a function of $\psi_{\mathrm{HWP}}$

The experimental data have been fitted with a linear function, whose slope is 4.04 (red continuous line). The theoretical curve (see Eq. (19)), whose slope is 4 , is the dashed blue line. One can see the good agreement between the experimental results and theory. Due to experimental uncertainties, the experimental data are slightly spread along the linear fit. The related standard deviation is $3.9^{\circ}$. Let us notice that the orientation of the HWP is experimentally known with a $1^{\circ}$-precision, and thus

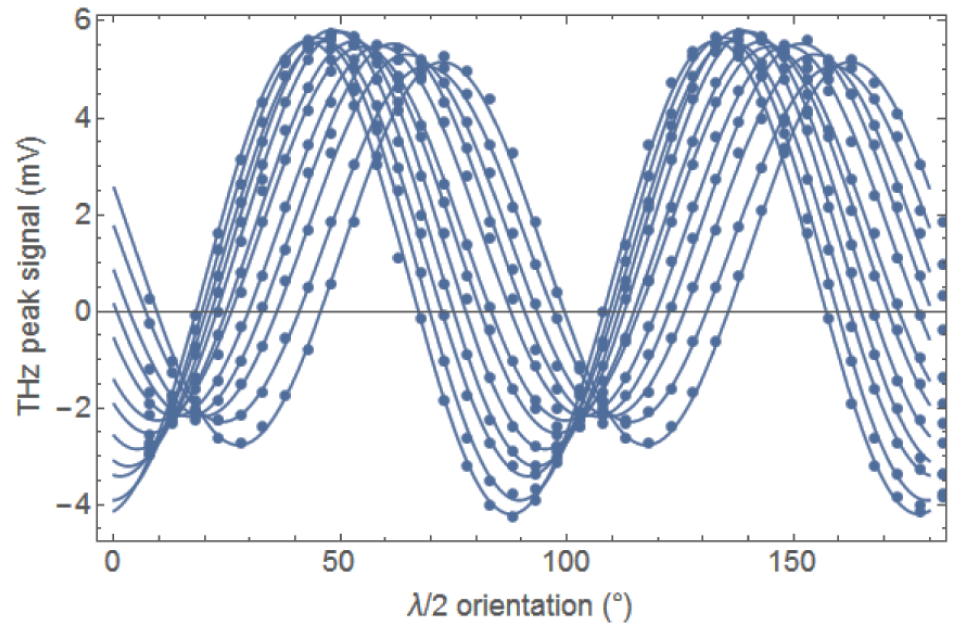

Fig. 3. $\mathrm{THz}$ peak signal as a function of the HWP orientation $\psi_{\mathrm{HWP}}$. Each curve corresponds to one orientation $\alpha$ of the $\mathrm{THz}$ receiver. 


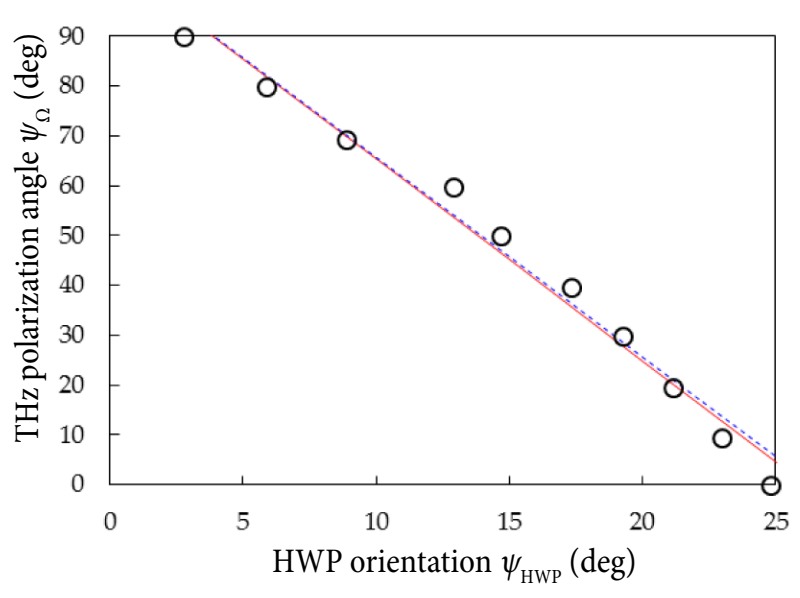

Fig. 4. THz polarization angle $\psi_{\Omega}$ as a function of the HWP orientation $\psi_{\text {HWP }}$ Open circles are experimental data. The red (online) continuous line is the best linear fit and the dashed blue (online) line is the theoretical curve.

the resulting imprecision of the $\mathrm{THz}$ polarization should be 4 times this value, i.e. $4^{\circ}$. The $\mathrm{THz}$ field vector deduced from both Figs. 3 and 4 is depicted in Fig. 5. Only one vector is drawn for the sake of legibility, but the full circles correspond to the end of each vector. The dashed lines give the uncertainty.

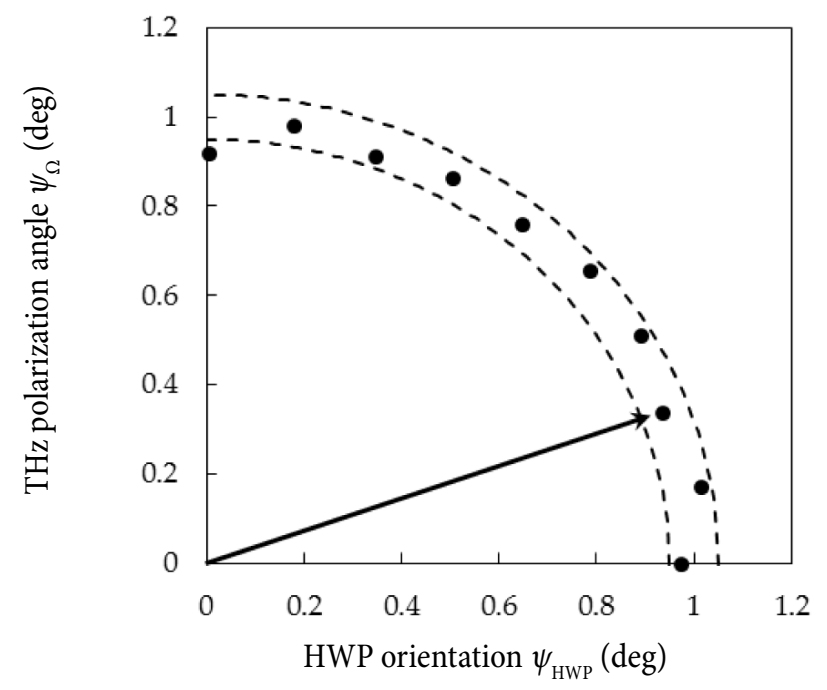

Fig. 5. End of each measured $\mathrm{THz}$ field vector (open circles). The vector for $\psi_{\Omega}=20^{\circ}$ is drawn. The dashed lines give the uncertainty.

\subsection{THz-TDS set-up with EO antennas for polarimetric studies}

The results described in the previous subsection have been exploited to generate various $\mathrm{THz}$ polarization states. In addition, the $\mathrm{THz}$ beam is de- tected and analysed with EO antennas that include a [111] ZnTe crystal. The optical source is the amplified laser system, whose huge power allows us to implement a detection scheme less efficient, but easier to adjust than the classical one. The $\mathrm{THz}$ emission is achieved as described in the previous paragraph. At the detector, we employ a [111] ZnTe crystal, and the laser probe beam is now circularly polarized instead of being linearly polarized. It allows us to get a detection signal whatever the orientation $\gamma$ of the ZnTe crystal, at the expense of a lower signal. After having crossed the ZnTe crystal, the probe beam is split in two beams thanks to a non-polarizing beam splitter. These two beams are respectively analysed along two eigen-axes, corresponding to the balanced measurement of $x$ and $y$ components of the THz field. The modulation induced by these two components of the $\mathrm{THz}$ field is acquired thanks to balanced photodiodes and a lock-in amplifier. The measured waveforms, corresponding to linearly and elliptically $\mathrm{THz}$ beams, are given in Fig. 6 .

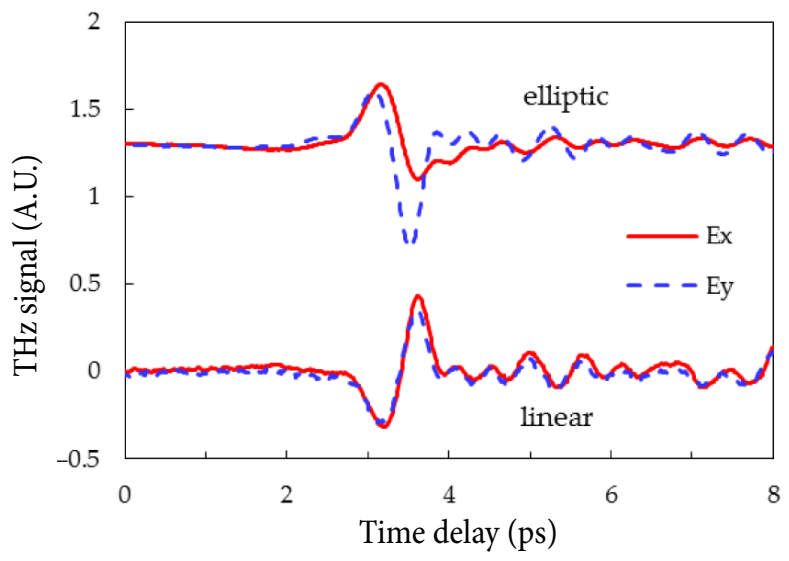

Fig. 6. THz waveforms for a linearly polarized signal (lower curve) and an elliptical one (upper curve, vertically shifted by 1.3 for the sake of legibility). The red (online) curves correspond to the $x$ component of the $\mathrm{THz}$ fields, and the dashed blue (online) ones to the $y$ component.

The experimental signal-to-noise ratio of these waveforms reaches $45 \mathrm{~dB}$. The frequency bandwidth associated with these transient signals spreads up to $4 \mathrm{THz}$. A crucial issue related to this experimental procedure concerns the accuracy of the phase measurement, which actually leads to the $\mathrm{THz}$ field polarization. The temporal position 
of each recorded data in the $E_{\Omega x}$ and $E_{\Omega y}$ waveforms depends mostly on the delay line (equivalent time scale) and on the exact polarization direction measured on each path of the two-beams scheme (i.e. the precision of the eigen-axes). This latter one leads to the major uncertainty because of the accuracy of the HWP orientation, which defines the $x$ and $y$ directions with eventually an uncertainty $\Delta \psi_{\Omega}=4^{\circ}$. Actually, as the $\mathrm{THz}$ field polarization angle is $\psi_{\Omega}=\operatorname{atan}\left(E_{\Omega y} / E_{\Omega x}\right)$, any error in the measurement of $E_{\Omega x}$ and $E_{\Omega y}$ gives

$$
\Delta \psi_{\Omega}=\frac{E_{\Omega y} \Delta E_{\Omega x}+E_{\Omega x} \Delta E_{\Omega y}}{E_{\Omega x}^{2}+E_{\Omega y}^{2}} .
$$

Assuming $\Delta E_{\Omega x} / E_{\Omega x}=\Delta E_{\Omega y} / E_{\Omega y}=1 \%, \Delta \psi_{\Omega}<0.6^{\circ}$. This value remains much smaller than the one coming from the HWP.
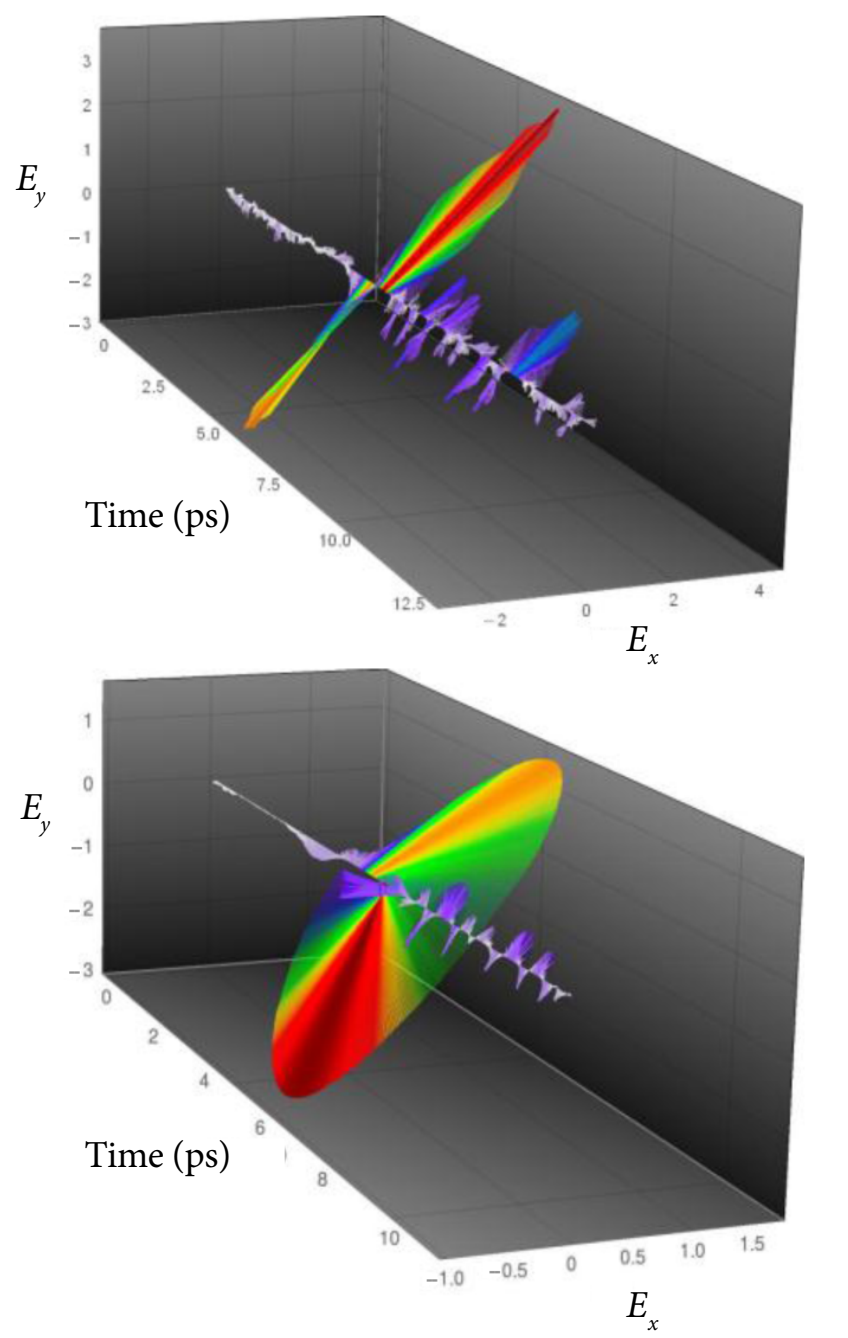

Fig. 7. 3D-plots of the $\mathrm{THz}$ field vectors versus time. Upper plot: linear THz polarization; lower plot: elliptical polarization.
A vectorial representation of the $\mathrm{THz}$ vector, corresponding to the waveforms of Fig. 6 , is plotted versus time in Fig. 7. The linear (Fig. $7(a)$ ) and elliptical (Fig. $7($ b)) polarizations are clearly evidenced.

\section{TPA effect in [111] crystals}

Amplified femtosecond laser systems could be employed to achieve a large signal-over-noise ratio when dealing with the OR generation of $\mathrm{THz}$ signals, because the generated $\mathrm{THz}$ field is proportional to the laser intensity. Unfortunately, the huge peak power induces other nonlinear effects, like two-photon absorption (TPA). When TPA occurs, free carriers are excited in the conduction band of the crystal. This results in two effects: 1) the laser intensity decreases through the crystal, which leads to a weaker $\mathrm{THz}$ generation; 2) the $\mathrm{THz}$ beam is itself absorbed by the free carriers. TPA is a third order nonlinear process that involves two photons of the laser beam, and a third one which is either a laser photon in the case of TPA of the laser light, or a THz photon when looking at TPA of the THz beam. Both effects make the OR generation efficiency saturating at high pump power.

\subsection{Simple modelling of the TPA effect}

Using the same procedure as above (see Eq. (4)), and in the undepleted pump beam approximation, the propagation equations of the laser and $\mathrm{THz}$ fields are

$$
\begin{aligned}
& \frac{\partial^{2} \vec{E}_{\omega}}{\partial z^{2}}+\frac{\omega^{2}}{c^{2}}\left(\widetilde{\varepsilon}_{\omega}+\vec{\chi}_{\omega}^{(3)}: \vec{E}_{\omega} \cdot \vec{E}_{\omega}^{*}\right) \vec{E}_{\omega}=0, \\
& \frac{\partial^{2} \vec{E}_{\Omega}}{\partial z^{2}}+\frac{\Omega^{2}}{c^{2}}\left(\widetilde{\varepsilon}_{\omega}+\vec{\chi}_{\Omega}^{(3)}: \vec{E}_{\omega} \cdot \vec{E}_{\omega}^{*}\right) \vec{E}_{\Omega} \\
& =-\frac{\Omega^{2}}{c^{2}} \vec{\chi}_{\Omega}^{(2)}: \vec{E}_{\omega} \cdot \vec{E}_{\omega}^{*},
\end{aligned}
$$

with $\quad \vec{\chi}_{\omega}^{(3)}=\ddot{\chi}^{(3)}(\omega, \omega,-\omega, \omega), \quad \ddot{\chi}_{\Omega}^{(3)}=\ddot{\chi}^{(3)}(\Omega, \omega,-\omega, \Omega), \quad$ and $\bar{\chi}_{\Omega}^{(2)}=\ddot{\chi}^{(2)}(\Omega, \omega,-\omega)$. In zinc-blende crystals, only the following elements of the nonlinear tensors are not null:

$$
\chi_{I J K L}^{(3)} \equiv \chi_{1}^{(3)}, \chi_{I I J J}^{(3)} \equiv \chi_{2}^{(3)}, \chi_{I J I J}^{(3)} \equiv \chi_{3}^{(3)}, \chi_{I J J I}^{(3)} \equiv \chi_{4}^{(3)} .
$$


Moreover, because TPA is induced by the photons of the same pump laser beam, the two central indices in (32) may be permuted, which results in

$$
\chi_{2}^{(3)}=\chi_{3}^{(3)}
$$

Equation (30) exhibits an analytical solution within some approximations, but Eq. (31) must be solved numerically. Let us first treat Eq. (30), following a calculation published by Bepko [21]. We set $\vec{E}_{\omega}=\vec{E}_{\omega}(z) \mathrm{e}^{\mathrm{in}_{\omega} \frac{\omega}{c} z}$ and we neglect the second derivative in (30). We obtain for each component $\widetilde{E}_{\omega i}(z)$ of $\vec{E}_{\omega}(z)$

$$
\begin{aligned}
& 2 \mathrm{i} \frac{\omega}{c} n_{\omega} \frac{\partial \widetilde{E}_{\omega i}(z)}{\partial z}+\left(2 \mathrm{i} n_{\omega}-\kappa_{\omega}\right) \kappa_{\omega} \widetilde{E}_{\omega i}(z) \\
& =-\sum_{j k l}\left(\vec{\chi}_{\omega i j k l}^{(3)}: \widetilde{E}_{\omega j}(z) \cdot \widetilde{E}_{\omega k}^{*}(z) \cdot \widetilde{E}_{\omega l}(z)\right) .
\end{aligned}
$$

In [111] cubic crystals, the nonlinear term in (34) is first calculated in the crystal frame and then in the laboratory frame, as previously explained. One obtains

$$
\begin{aligned}
& \vec{\chi}_{\omega}^{(3)}: \vec{E}_{\omega}(z) \cdot \vec{E}_{\omega}^{*}(z) \cdot \vec{E}_{\omega}(z)=\frac{\left(\widetilde{E}_{\omega}(z)\right)^{3}}{2} \\
& \times\left(\begin{array}{c}
\left(\chi_{1}^{(3)}+3 \chi_{2}^{(3)}\right) \cos \psi \\
\left(\chi_{1}^{(3)}+3 \chi_{2}^{(3)}\right) \sin \psi \\
\frac{\sqrt{2}}{3}\left(\chi_{1}^{(3)}-3 \chi_{2}^{(3)}\right)\left(3 \cos ^{2} \psi-\sin ^{2} \psi\right) \cos \psi
\end{array}\right) .
\end{aligned}
$$

$\widetilde{E}_{\omega i}(z)$ is the complex magnitude of $\vec{E}_{\omega}(z)$. Only the $x$ and $y$ components in (35) must be considered because the $z$ component of the $E$ field does not propagate in air. As they are equal to the projection of $\frac{1}{2}\left(\widetilde{E}_{\omega}(z)\right)^{2}\left(\chi_{1}^{(3)}+3 \chi_{2}^{(3)}\right) \vec{E}_{\omega}(z)$ along, respectively, $x$ and $y$, the TPA phenomenon is isotropic, and Eq. (34) simply leads to

$$
\begin{aligned}
& 2 \mathrm{i} \frac{\omega}{c} n_{\omega} \frac{\partial \widetilde{E}_{\omega}(z)}{\partial z}+\left(2 \mathrm{i} n_{\omega}-\kappa_{\omega}\right) \kappa_{\omega} \widetilde{E}_{\omega}(z) \\
& =-\frac{\chi_{1}^{(3)}+3 \chi_{2}^{(3)}}{2}\left(\widetilde{E}_{\omega}(z)\right)^{3} .
\end{aligned}
$$

Then, one easily derives the expression of the intensity $I_{\omega}(z)$ of the laser beam after propagating over a distance $z$ in the crystal [21]:

$$
\begin{aligned}
& \frac{\mathrm{d} I_{\omega}(z)}{\mathrm{d} z}+\alpha_{\omega} I_{\omega}(z)=-\beta_{\omega} I_{\omega}^{2}(z) \\
& \Rightarrow I_{\omega}(z) \approx \frac{I_{\omega}(0) \mathrm{e}^{-\alpha_{\omega} z}}{1+\frac{\beta}{\alpha_{\omega}} I_{\omega}(0)\left(1-\mathrm{e}^{-\alpha_{\omega} z}\right)} \approx \frac{I_{\omega}(0)}{1+\beta I_{\omega}(0) z} .
\end{aligned}
$$

This approximated solution is obtained if the nonlinear effect is weak, i.e. $\beta_{\omega}=Z_{o} \frac{\omega}{c} \frac{1}{n_{\omega}^{2}} \operatorname{Im}\left(\chi_{1}^{(3)}+3 \chi_{2}^{(3)}\right)$ is small. This is the well-known expression of the saturation of the crystal optical transmission due to the TPA phenomenon.

Solving Eq. (31) is more difficult. However, in zinc-blende crystals, we have already shown (see Eq. (9)) that THz generation by OR does not depend on the laser polarization angle $\psi$ when the crystal cut is [111], as well as TPA of the laser beam (Eq. (35)). Therefore, what could induce a $\psi$-dependence (i.e. anisotropy) in THz generation is only the TPA term of the $\mathrm{THz}$ signal in Eq. (31), i.e. $\vec{\chi}_{\Omega}^{(3)}: \vec{E}_{\omega} \cdot \vec{E}_{\omega}^{*} \cdot \vec{E}_{\Omega}$. Without solving rigorously (31), let us treat simply the anisotropy of the generated $\mathrm{THz}$ signal [38]. The propagation coefficient of the $\mathrm{THz}$ field appears in the second term in the left part of (31), namely $\frac{\Omega^{2}}{c^{2}}\left(\widetilde{\varepsilon}_{\Omega}+\vec{\chi}_{\Omega}^{(3)}: \vec{E}_{\omega} \cdot \vec{E}_{\omega}^{*}\right) \vec{E}_{\Omega}$. The absorption of the $\mathrm{THz}$ field corresponds to the imaginary part of the square-root of this term:

$$
\vec{\alpha}_{\Omega}\left(\vec{E}_{\omega}\right)=2 \frac{\Omega}{c} \operatorname{Im} \sqrt{\widetilde{\varepsilon}_{\Omega}+\frac{\vec{\chi}_{\Omega}^{(3)}: \vec{E}_{\omega} \cdot \vec{E}_{\omega}^{*} \cdot \vec{E}_{\Omega}}{\vec{E}_{\Omega}}} .
$$

Let us notice that, because of the tensorial TPA term in (38), the coefficient of absorption is anisotropic. If this nonlinear term is small as compared to $\widetilde{\varepsilon}_{\Omega}$, we can take the first-order series expansion of (38):

$$
\begin{aligned}
& \vec{\alpha}_{\Omega}\left(\vec{E}_{\omega}\right) \approx 2 \frac{\Omega}{c} \operatorname{Im}\left(\widetilde{n}_{\Omega}\left(1+\frac{\vec{\chi}_{\Omega}^{(3)}: \vec{E}_{\omega} \cdot \vec{E}_{\omega}^{*} \cdot \vec{E}_{\Omega}}{2 \widetilde{\varepsilon}_{\Omega} \vec{E}_{\Omega}}\right)\right) \\
& \approx \alpha(\Omega)+\frac{\Omega}{c} \frac{\operatorname{Im}\left(\vec{\chi}_{\Omega}^{(3)}\right): \vec{E}_{\omega} \cdot \vec{E}_{\omega}^{*} \cdot \vec{E}_{\Omega}}{n(\Omega) \vec{E}_{\Omega}} .
\end{aligned}
$$

Here we have supposed that the imaginary part of $\widetilde{n}_{\Omega}=\sqrt{\widetilde{\varepsilon}_{\Omega}}$ is small as compared to the real part $n_{\Omega}$. It follows that the change of absorption induced by the TPA phenomenon writes

$$
\begin{aligned}
& \vec{\alpha}_{\mathrm{TPA}, \Omega} \approx \frac{\Omega}{c} \frac{1}{n_{\Omega}} \frac{\operatorname{Im}\left(\ddot{\chi}_{\Omega}^{(3)}\right): \vec{E}_{\omega} \cdot \vec{E}_{\omega}^{*} \cdot \vec{E}_{\Omega}}{\vec{E}_{\Omega}}, \\
& \Rightarrow \alpha_{\mathrm{TPA}, \Omega, i} \\
& \propto \operatorname{Im}\left(\chi_{1}^{(3)} E_{\omega, 1}^{2} E_{\Omega, i}+\chi_{2}^{(3)} E_{\omega, i} \sum_{j}\left(1-\delta_{i j}\right) E_{\omega, j} E_{\Omega, j}\right) .
\end{aligned}
$$


When taking into account the [111] orientation of the crystal and entering in (40) the field components defined in the laboratory frame, as explained in the first part of this paper, we get

$$
\Delta_{\mathrm{TPA}} \vec{E}_{\Omega, \text { lab }} \propto-E_{0}^{4}\left(\begin{array}{c}
A \cos 2 \psi+B \cos 4 \psi \\
B \sin 4 \psi-A \sin 2 \psi \\
C
\end{array}\right),
$$

with

$$
\left\{\begin{array}{c}
A=\chi_{1}^{(3)}+4 \chi_{2}^{(3)}+5 \chi_{4}^{(3)}, \\
B=\chi_{1}^{(3)}+4 \chi_{2}^{(3)}-\chi_{4}^{(3)}, \\
C=3 \sqrt{2} \chi_{4}^{(3)} .
\end{array}\right.
$$

\subsection{Experimental observation of the TPA effect}

When looking precisely at the representation of the $\mathrm{THz}$ vector versus time in Fig. $7(\mathrm{~b})$, the $\mathrm{THz}$ polarization is not perfectly circular, but elliptic. However, the polarization of the pumping laser beam is almost perfectly circular. As explained above, this is the signature of the TPA effect. For a better evaluation of this anisotropy, we analyse the OR efficiency of $\mathrm{THz}$ generation as a function of the orientation of a linearly polarized pump beam. We use two different detectors, namely a Schottky diode and an EO antenna detection. Both detectors are sensitive to only one direction of $\mathrm{THz}$ polarization. If there is no TPA effect, the $\mathrm{THz}$ signal is constant versus $\psi_{\mathrm{HWP}}$ and polarized along $2 \psi=4 \psi_{\mathrm{HWP}}$. The detected signal is proportional to the projection of the $\mathrm{THz}$ field along the direction of sensitivity of the detector and should vary as $\cos \left(4 \psi_{\mathrm{HWP}}\right)$ (dashed curve in Fig. 8). But the recorded data in Fig. 8 do not follow this theoretical curve. Two successive angular maxima differ by more than $20 \%$. The vertical component of the field is thus much more attenuated than the horizontal component as predicted by relation (41). The curve (continuous line in Fig. 8) calculated with this expression (41) fits very well the measured data. Let us notice that this effect was not observed before our publication [39], because studies are aiming at getting the strongest $\mathrm{THz}$ signal from ZnTe crystals, and thus mostly the [110] orientation is employed, for which the crystal anisotropy hides the TPA anisotropy effect.

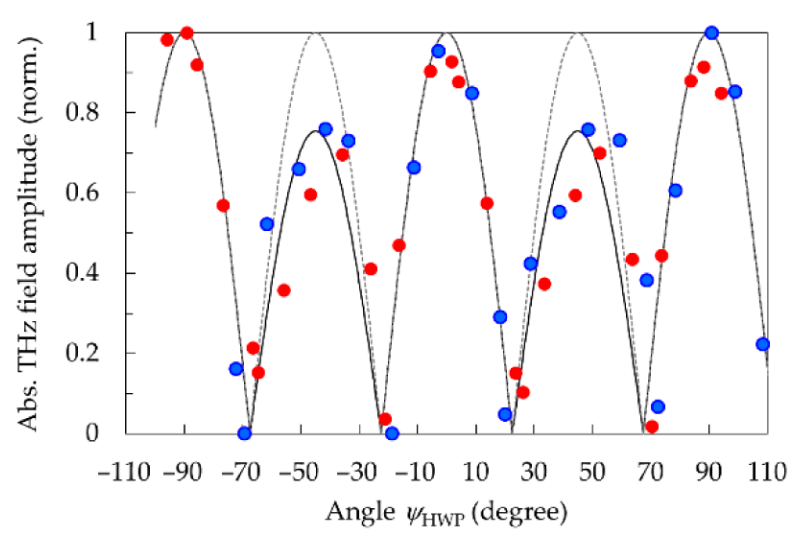

Fig. 8. THz peak value (normalized) as a function of $\psi_{\mathrm{HWP}}$ Smaller (red online) dots: data recorded with a Schottky diode. Bigger (blue online) dots: data recorded with an EO crystal. Black curve: fit using Eq. (41). Dashed gray curve: response without TPA effect.

\section{Conclusions}

We have shown that using [111] zinc blende crystals in a THz-TDS system makes it possible to easily generate and detect $\mathrm{THz}$ pulses with any polarization state. The great advantage is that no polarization component at $\mathrm{THz}$ frequencies is required. All the manipulation of the $\mathrm{THz}$ polarization is performed by adjusting the polarization of the laser pump and probe beams. Thus THz-TDS set-ups dedicated to polarimetric studies are easy to build up. They can be substituted to more complex TDS systems in which the polarization issue is, for example, addressed by rotating the antennas [40, 43], or by employing bi-polarization sensitive photoconducting switches [42, 43].

\section{Acknowledgements}

This research, and especially the work of Dr. F. Sanjuan, was partially supported by the French Research Agency (ANR) through the LabEx FOCUS ANR-11-LABX-0013 Project. G. Gaborit is also with Kapteos S.A.S., 73800 Sainte-Hélène du Lac, France.

\section{References}

[1] D.T. Emerson, The work of Jagadis Chandra Bose: 100 years of millimeter-wave research, IEEE Trans. Microw. Theory Techn. 45, 2267-2273 (1997). 
[2] E.D. Palik, History of far-infrared research I. The Rubens era, J. Opt. Soc. Am. 67, 857-865 (1977).

[3] E.F. Nichols and J.D. Tear, Short electric waves, Phys. Rev. 21, 587-610 (1923).

[4] K.P. Cheung and D.H. Auston, A novel technique for measuring far-infrared absorption and dispersion, Infrared Phys. 26, 23-27 (1986).

[5] E. Matsubara, M. Nagai, and M. Ashida, Coherent infrared spectroscopy system from terahertz to near-infrared using air plasma produced by $10-\mathrm{fs}$ pulses, J. Opt. Soc. Am. B 30, 1627-1630 (2013).

[6] A. Leitenstorfer, K.A. Nelson, K. Reimann, and K. Tanaka, Focus on nonlinear terahertz studies, New J. Phys. 16, 045016 (2014).

[7] A. Krotkus, Semiconductors for terahertz photonics applications, J. Phys. D 43, 273001 (2010).

[8] Q. Chen, M. Tani, Z. Jiang, and X.-C. Zhang, Electro-optic transceivers for terahertz-wave applications, J. Opt. Soc. Am. B 18, 823-831 (2001).

[9] H. Minamide, J. Zhang, R. Guo, K. Miyamoto, S. Ohno, and H. Ito, High-sensitivity detection of terahertz waves using nonlinear up-conversion in an organic 4-dimethylamino-N-methyl-4-stilbazolium tosylate crystal, Appl. Phys. Lett. 97, 121106 (2010).

[10]M.C. Hoffmann, K.-L. Yeh, J. Hebling, and K.A. Nelson, Efficient terahertz generation by optical rectification at $1035 \mathrm{~nm}$, Opt. Express 15, 11706 (2007).

[11]A. Schneider, M. Neis, M. Stillhart, B. Ruiz, R.U.A. Khan, and P. Günter, Generation of terahertz pulses through optical rectification in organic DAST crystals: theory and experiment, J. Opt. Soc. Am. B 23, 1822-1835 (2006).

[12]Q. Wu and X.-C. Zhang, Design and characterization of traveling-wave electrooptic terahertz sensors, IEEE J. Sel. Top. Quant. Electron. 2, 693700 (1996).

[13] H. Ito, K. Suizu, T. Yamashita, A. Awahara, and T. Sato, Random frequency accessible broad tunable terahertz-wave source using phase-matched 4 dimethylamino-N-methyl-4-stilbazolium tosylate crystal, Jpn. J. Appl. Phys. 46, 7321-7324 (2007).

[14]L. Duvillaret, S. Rialland, and J.-L. Coutaz, Electro-optic sensors for electric field measure- ments. I. Theoretical comparison among different modulation techniques, J. Opt. Soc. Am. B 19, 2692-2703 (2002).

[15]L. Duvillaret, S. Rialland, and J.-L. Coutaz, Electro-optic sensors for electric field measurements. II. Choice of the crystals and complete optimization of their orientation, J. Opt. Soc. Am. B 19, 2704-2715 (2002).

[16]G. Gaborit, J.-L. Coutaz, and L. Duvillaret, Vectorial electric field measurement using isotropic electro-optic crystals, Appl. Phys. Lett. 90, 241118 (2007).

[17]N.C. van der Valk, W.A. van der Marel, and P.C. Planken, Terahertz polarization imaging, Opt. Lett. 30, 2802-2804 (2005).

[18]F. Zernike, Jr. and P.R. Berman, Generation of far-infrared as a difference frequency, Phys. Rev. Lett. 15, 999-1001 (1965).

[19]D.W. Faries, K.A. Gehring, P.L. Richards, and Y.R. Shen, Tunable far-infrared radiation generated from the difference frequency between two ruby lasers, Phys. Rev. 180, 363-365 (1969).

[20]N. Van Tram and C.K.N. Patel, Free-carrier magneto-optical effects in far-infrared difference-frequency generation in semiconductors, Phys. Rev. Lett. 22, 464-466 (1969).

[21]F. Zernike, Temperature-dependent phase matching for far-infrared difference-frequency generation in InSb, Phys. Rev. Lett. 22, 931-933 (1969).

[22]J.M. Yarborough, S.S. Sussman, H.E. Puthoff, R.H. Pantell, and B.C. Johnson, Efficient tunable optical emission from $\mathrm{LiNbO}_{3}$ without a resonator, Appl. Phys. Lett. 15, 102-104 (1969).

[23]J. Morris and Y.R. Shen, Far-infrared generation by picosecond pulses in electrooptic materials, Opt. Commun. 3, 81-84 (1971).

[24]K.H. Yang, P.L. Richards, and Y.R. Shen, Generation of far-infrared radiation by picosecond light pulses in $\mathrm{LiNbO}_{3}$, Appl. Phys. Lett. 19, 320-322 (1971).

[25]A. Bonvalet, M. Joffre, J.-L. Martin, and A. Migus, Generation of ultrabroad band femtosecond pulses in the mid-infrared by optical rectification of 15 fs light pulses at $100 \mathrm{MHz}$ repetition rate, Appl. Phys. Lett. 67, 2907-2909 (1995). 
[26]P.Y. Han and X.-C. Zhang, Coherent, broadband mid infrared terahertz beam sensors, Appl. Phys. Lett. 73, 3049-3051 (1998).

[27]P.Y. Han and X.-C. Zhang, Free-space coherent broadband terahertz time-domain spectroscopy, Meas. Sci. Technol. 12, 1747-1756 (2001).

[28]N. Amer, W.C. Hurlbut, B.J. Norton, Y.-S. Lee, and T.B. Norris, Generation of terahertz pulses with arbitrary elliptical polarization, Appl. Phys. Lett. 87, 221111 (2005).

[29] M. Sato, T. Higuchi, N. Kanda, K. Konishi, K. Yoshioka, T. Suzuki, and M. Kuwata-Gonokami, Terahertz polarization pulse shaping with arbitrary field control, Nat. Phot. 7, 724-731 (2013).

[30]G. Gaborit, A. Biciunas, M. Bernier, and J.-L. Coutaz, Emitting and receiving terahertz vectorial antennas based on cubic electro-optic crystals, IEEE Trans. Terahertz Sci. Technol. 5, 828-835 (2015).

[31]J.A. Valdmanis, G. Mourou, and C.W. Gabel, Picosecond electro-optic sampling system, Appl. Phys. Lett. 41, 211-213 (1982).

[32]Q. Wu and X.-C. Zhang, Free-space electro-optic sampling of terahertz beams, Appl. Phys. Lett. 67, 3523-3525 (1995).

[33]P.U. Jepsen, C. Winnewisser, M. Schall, V. Schyja, S.R. Keiding, and H. Helm, Detection of $\mathrm{THz}$ pulses by phase retardation in lithium tantalate, Phys. Rev. E 53, R3052-R3054 (1996).

[34] A. Nahata, D.H. Auston, T.F. Heinz, and C. Wu, Coherent detection of freely propagating terahertz radiation by electro-optic sampling, Appl. Phys. Lett. 68, 150-152 (1996).

[35]N. Yasumatsu, and S. Watanabe, Precise real-time polarization measurement of terahertz electromagnetic waves by a spinning electro-optic sensor, Rev. Sci. Instrum. 83, 023104 (2012).
[36]N. Yasumatsu, A. Kasatani, K. Oguchi, and S. Watanabe, High-speed terahertz time-domain polarimeter based on an electro-optic modulation technique, App. Phys. Exp. 7, 092401 (2014).

[37]C.M. Morris, R.V. Aguilar, A.V. Stier, and N.P. Armitage, Polarization modulation timedomain terahertz polarimetry, Opt. Express 20, 12303-12317 (2012).

[38]N. Nemoto, T. Higuchi, N. Kanda, K. Konishi, and M. Kuwata-Gonokami, Highly precise and accurate terahertz polarization measurements based on electro-optic sampling with polarization modulation of probe pulses, Opt. Express 22, 17915-17929 (2014).

[39]F. Sanjuan, G. Gaborit, and J.-L. Coutaz, Influence of two-photon absorption anisotropy on terahertz emission through optical rectification in zinc-blende crystals, J. Infrared Millim. Terahertz Waves 39, 378 (2018).

[40]F. Garet, L. Duvillaret, and J.-L. Coutaz, Evidence of frequency dependent $\mathrm{THz}$ beam polarization in time-domain spectroscopy, repercussions on anisotropic materials characterization, Proc. SPIE 3617, 38 (1999).

[41]C.D.W. Mosley, M. Failla, D. Prabhakaran, and J. Lloyd-Hughes, Terahertz spectroscopy of anisotropic materials using beams with rotatable polarization, Sci. Rep. 7, 12337 (2017).

[42]E. Castro-Camus, J. Lloyd-Hughes, M.B. Johnston, M.D. Fraser, H.H. Tan, and C. Jagadish, Polarization-sensitive terahertz detection by multi contact photoconductive receivers, Appl. Phys. Lett. 86, 254102 (2005).

[43]E. Castro-Camus and M.B. Johnston, Extraction of the anisotropic dielectric properties of materials from polarization-resolved terahertz timedomain spectra, J. Opt. A 11, 105206 (2009).

\title{
ANTROSIOS EILĖS NETIESINIAI OPTINIAI VYKSMAI [111] KUBINIUOSE KRISTALUOSE TERAHERCINEI OPTOELEKTRONIKAI
}

\author{
G. Gaborit, F. Sanjuan, J.-L. Coutaz
}

Savojos Monblano universitetas, Šamberi, Prancüzija 